\title{
THE PROGNOSIS IN RHEUMATOID ARTHRITIS IN CHILDHOOD
}

\author{
BY \\ THOMAS COLVER, M.B., M.R.C.P. \\ (From the Hospital for Sick Children, Great Ormond Street.)
}

Chronic polyarthritis in childhood is a disease of considerable frequency. In the medical wards of the Hospital for Sick Children, Great Ormond Street, sixty-nine cases have been treated since the war. There are additional outpatient cases which are not admitted to the wards and it is estimated that a new case may be seen at the hospital every twelve weeks.

In the wards the clinical picture of the acute phase is familiar, but apart from certain cases with early recovery the end results are seIdom seen, so prolonged is the course of the disease. The institutions for chronic rheumatoid arthritis collect the most severely crippled but these form a minority. The fairest assessment of prognosis is attained by a follow-up enquiry of a consecutive hospital series and for this purpose the post-war cases have been taken.

\section{The cases under review}

The series of sixty-nine is depicted in fig. 1 where the cases are arranged according to the age of onset. Two cases are seen to have begun in the first year of life, fourteen cases in the third year with a maximum incidence in the pre-school period between two and five years. The sex incidence is also indicated. Sixty per cent. of the patients were girls.

Follow-up results. The results of the follow-up enquiry are shown in fig. 2. Enquiries were successful in forty-nine cases (twenty cases were untraced). Of these, twelve are known to have died, eighteen are still active and nineteen are quiescent. The criteria of quiescence have been an absence of joint pain and tenderness for a period of not less than two years, and a blood sedimentation rate within normal limits. In no instance have symptoms recurred after a quiescent period of eighteen months.

The fatal cases. A recognition of the considerable mortality was the first finding of importance (fig. 2). Twelve of the patients followed have died and in each case before reaching adult age. Each fatal case had first developed the condition under five years of age and with one exception each one had died within three years of the onset. The disease is thus most dangerous in the acute stages in the young children.

Figure 1 shows that forty-two of the cases developed symptoms in the first five years of life. Of these, twelve are dead-a mortality of nearly thirty per cent. at this period. The disease is thus most fatal in the years when it is most frequent. 
ARCHIVES OF DISEASE IN CHILDHOOD

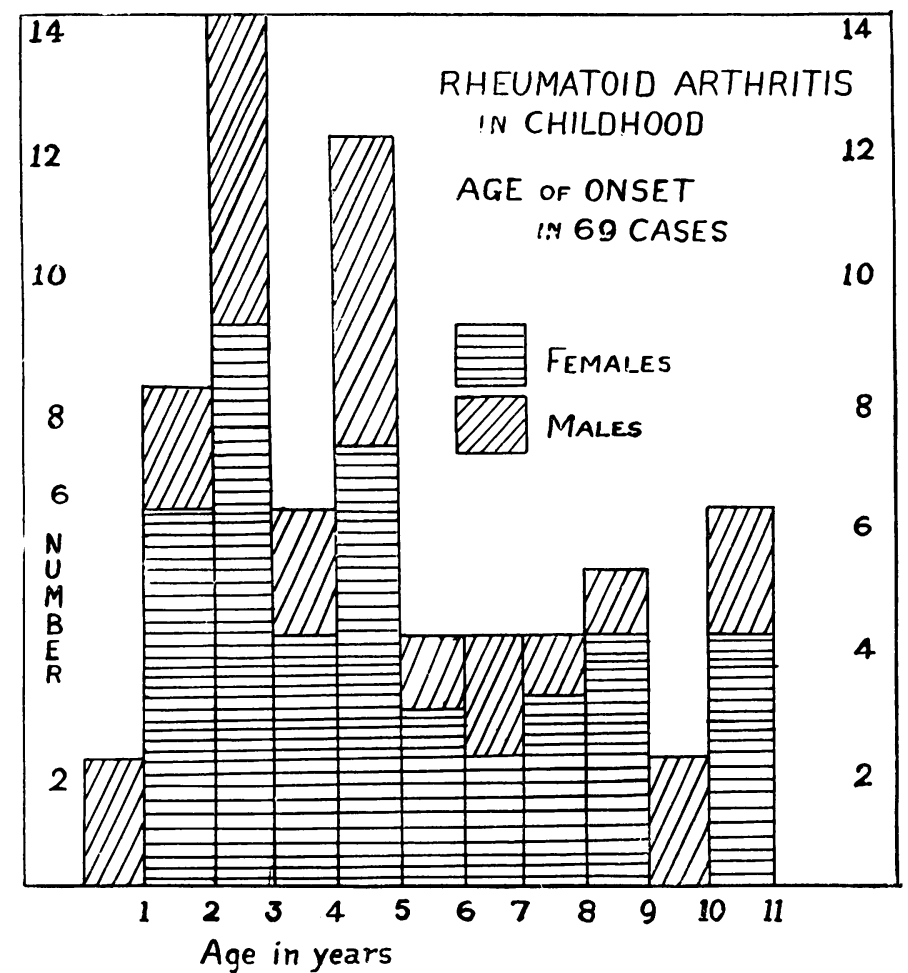

FIg. 1.

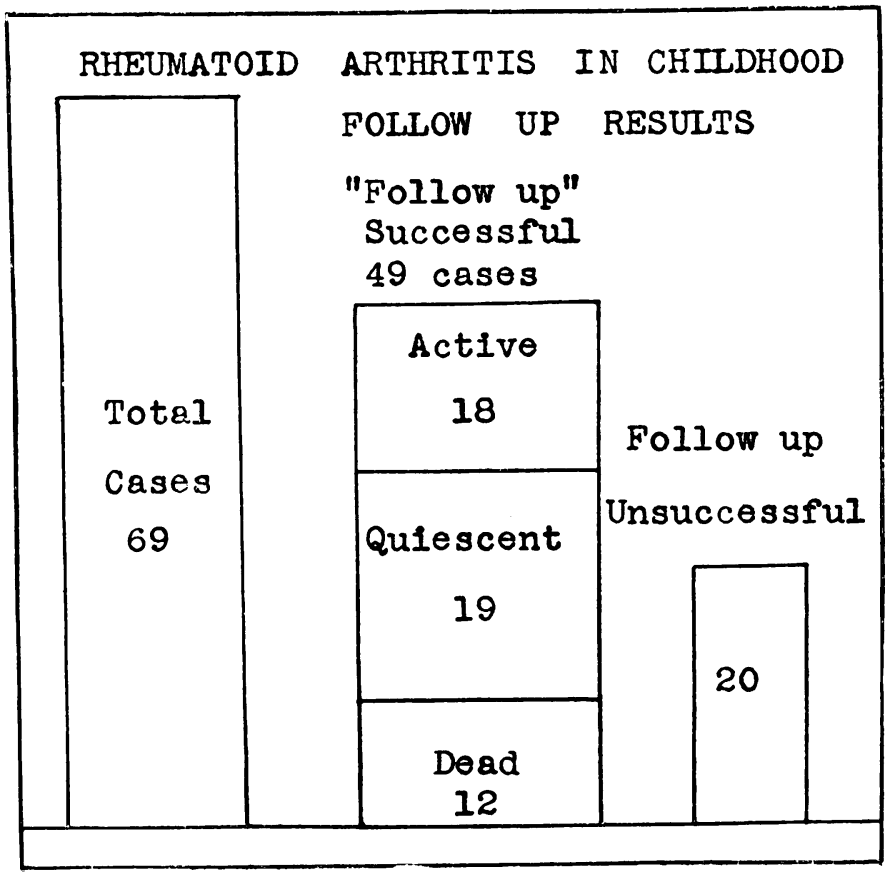

FIr. 2. 
When however, the disease begins after the age of five the prognosis as regards life is good. There is no record of the death of any such patient. Again if any patient (even the youngest) survives the first three years the prognosis as regards survival is good.

Cause of death in the fatal cases. Five patients died in hospital. Autopsies were performed in four of these. In all cases there was a progressive emaciation and anaemia, the patient gradually being reduced to an almost prostrate condition. In two cases there was a terminal streptococcal septicaemia (haemolytic), in one a large renal calculus and heavy urinary infection. A fourth showed advanced amyloid disease and extreme pericardial adhesions. In the fifth the autopsy revealed no finding of note apart from extreme emaciation and moderate pericardial adhesions. Pneumonia has been cited as the cause of death in most of the cases that died elsewhere.

The quiescent cases. Nineteen of the series are now quiescent. They have no pain and have lost the emaciation and anaemia characteristic of the active stages. A proportion of them show a varying amount of crippling, but when examining these older patients one was impressed by the frequency

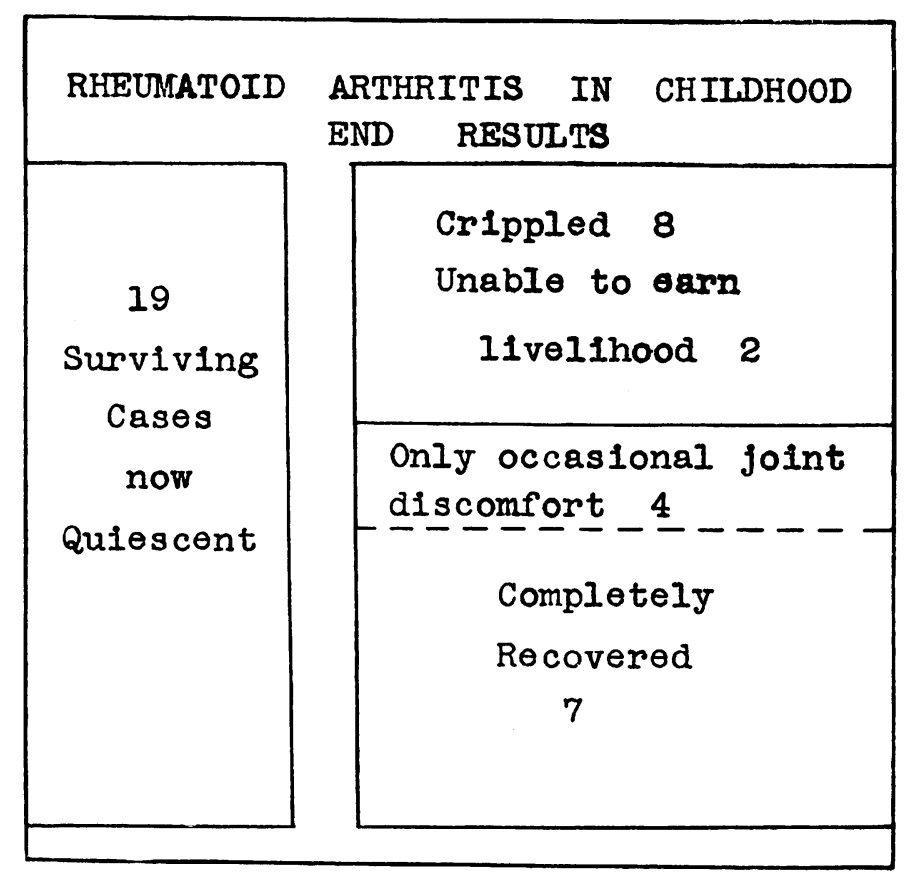

Fig. 3.

of complete recovery and by the relative infrequency of gross crippling. Crippling when it occurred was usually confined to two, three or four joints, the elbows and wrists being most frequently affected.

The details of these older patients are indicated in fig. 3. With two exceptions every patient can earn a livelihood. With one exception every patient can walk. Seven of the nineteen have completely recovered and a further four patients have recovered to all intents and purposes but full passive movement in one or two joints occasions slight discomfort. Eight old cases show some crippling but in only two of these is the ability to earn 
a livelihood lost. Of the patients earning a livelihood the most severely incapacitated has limited movement in four joints. One elbow and one finger are ankylosed in flexion and there is partial limitation in both wrists.

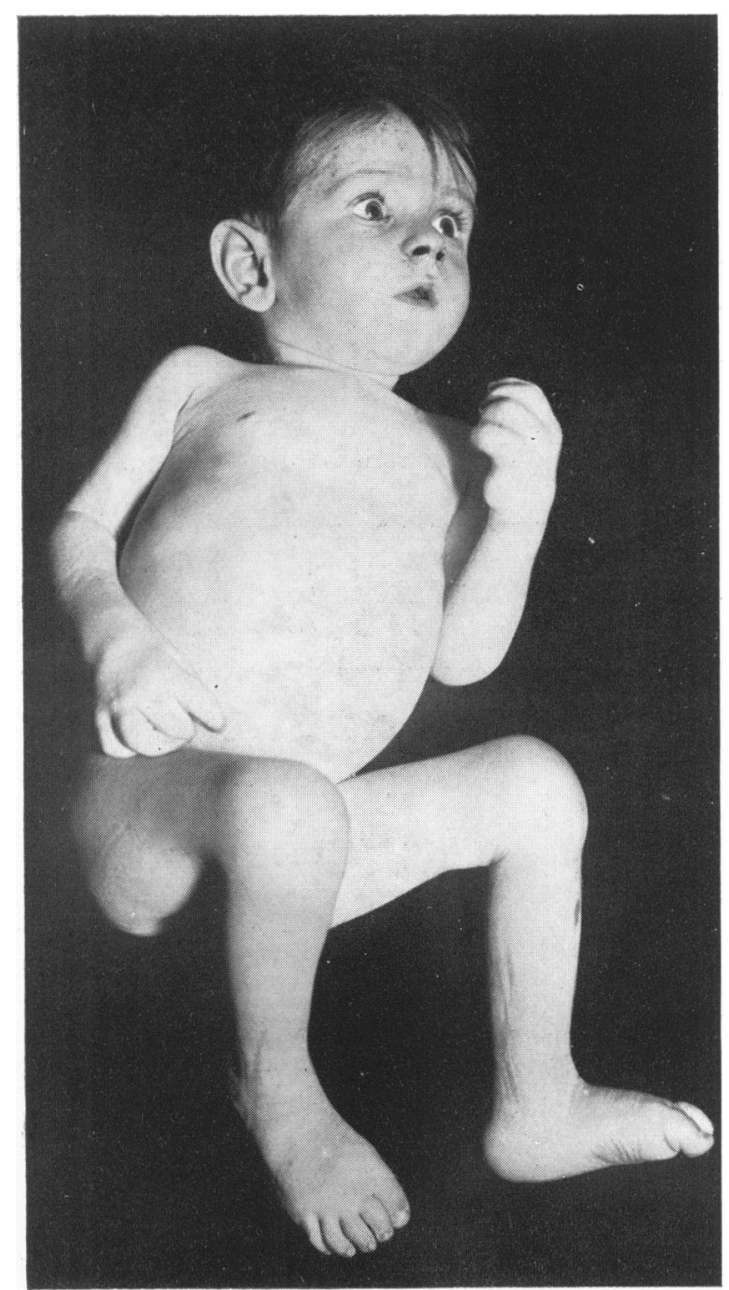

FIG. 4 .

(By courtesy of Dr. D. Paterson.)

Rheumatoid arthritis in infancy. The youngest case in the series. Symptoms began at six weeks of age and death occurred at nine months.

The duration of activity and its influence on the prognosis. Thirty-seven surviving cases have been examined; these include the active and quiescent cases indicated in fig. 2 and in no case has the duration of activity exceeded seven years. The disease thus appears to be self limited and in a given case one feels justified in predicting the cessation of activity within a maximum period. 
In the nineteen quiescent cases the average period of activity proved to be nearly five years. The duration of the active phase is one of the most important factors in estimating the prognosis and apparently three years of activity is the critical period. If after three years the disease is active the risk of a fatal outcome is negligible but the chance of complete

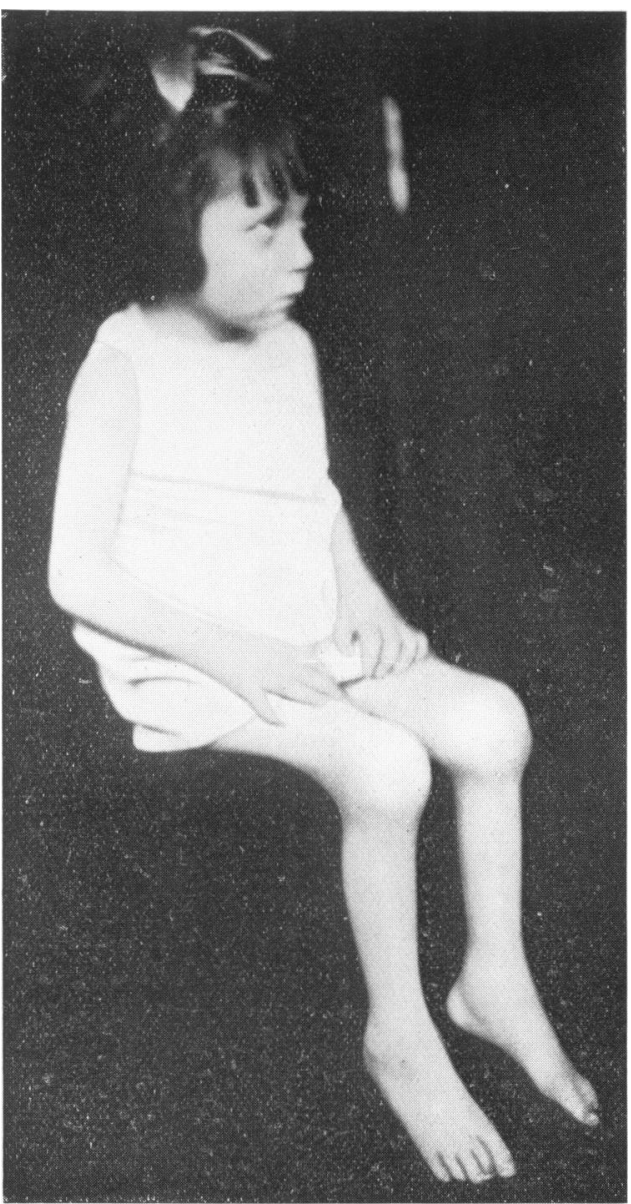

FIG. 5^.

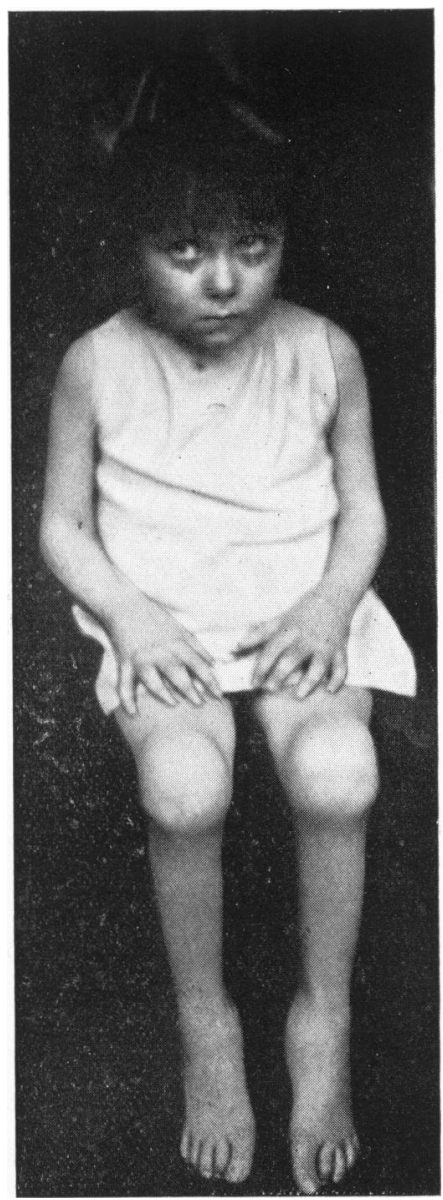

Fig. 5B.

(By courtesy of Dr. R. Hutchison.)

Rheumatoid arthritis in childhood. At this period the patient was five years of age. The disease was active for three years and was followed by complete recovery. (See Fig. $6 \mathrm{~A}$ and $\mathrm{B}$ ).

reccvery of function has almost disappeared. Eleven of the twelve deaths occurred within the first three years when constitutional upset is most severe. In the cases which completely recovered the disease ran a relatively short course, never longer than three years (twenty months was the shortest duration) and the end results also show that no case active for more than three years has completely recovered, 


\section{The treatment of foci of infection}

Exacerbations of the disease are frequently related to infections, particularly of the naso-pharynx. Results after the treatment of septic foci have however been discouraging. So prolonged is the disease and so great the wish to leave no stone unturned that operations for the removal of possible foci of

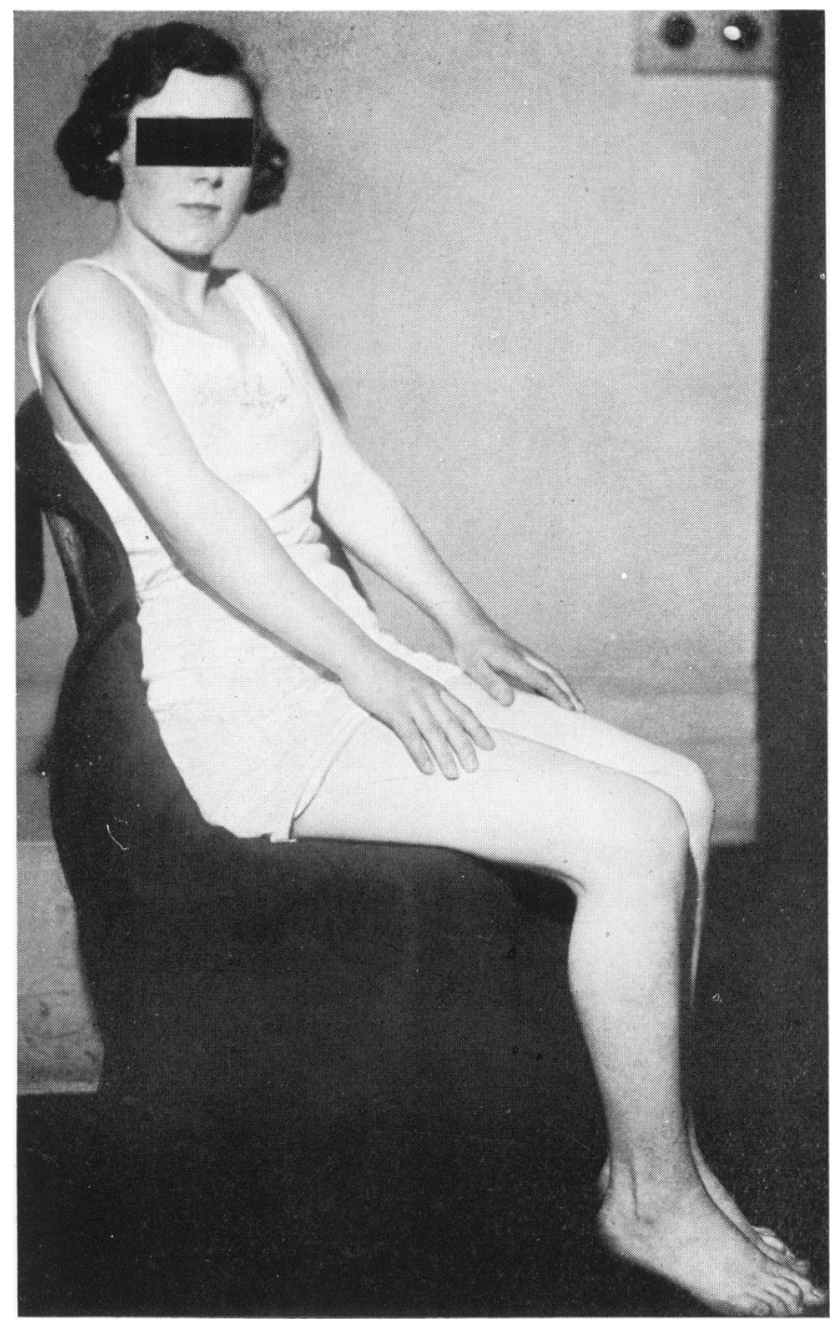

Fig. 6A.

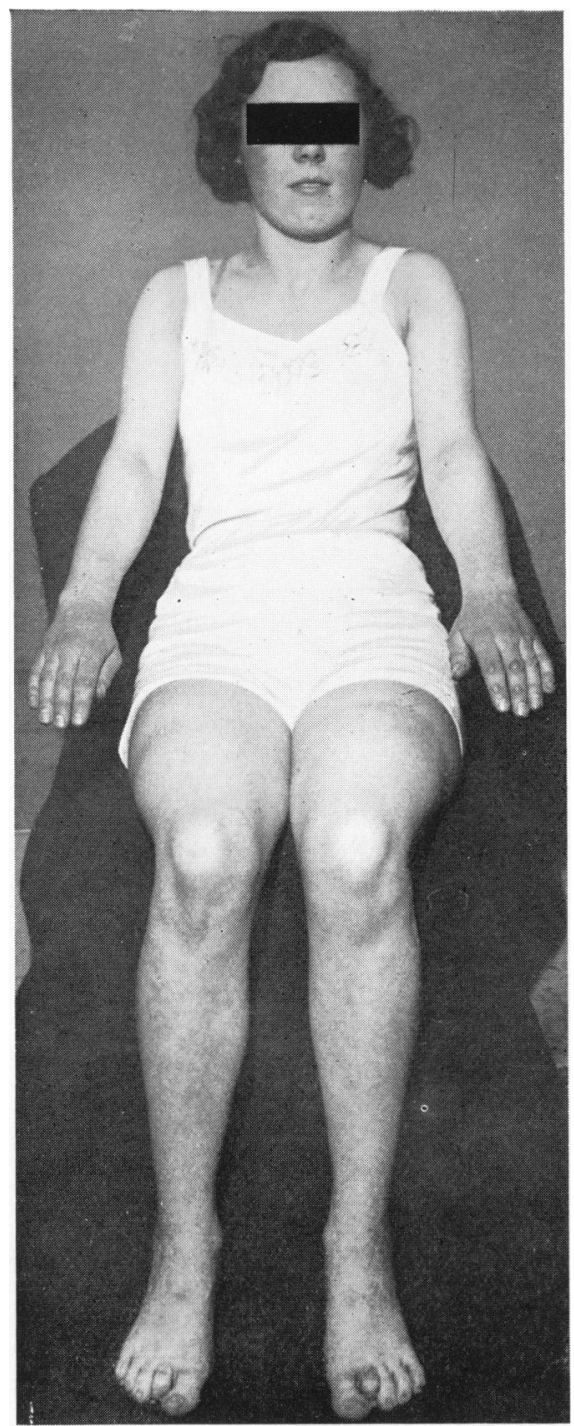

FIG. 6в. 
the relation of definite focal sepsis to the nnset of symptoms was so close that a special significance was difficult to refute.

In the first, symptoms began a few days after the removal of a grossly infected and gangrenous appendix; twelve months later exacerbations of the arthritis are still occurring. In one case symptoms began four days after the extraction of a carious tooth. There was an associated alveolar abscess. The subsequent extraction of a similarly infected tooth was followed by an exacerbation of symptoms. The active stage of the disease lasted two-and-a-half years. The patient is now symptom free. Recovery will probably be complete. In three cases the patients before the onset of symptoms presented that gross type of dental caries in which all the teeth are eroded almost to the gums. The teeth were removed in each case. One patient eventually died at the height of the disease eighteen months after the onset. In the second the disease is still active after three-and-ahalf years. The third has not been traced. In two cases symptoms followed severe tonsillitis. Recurrence of the tonsillitis was followed in each case by exacerbations and tonsil enucleations were performed. One patient died after two-and-a-half years. The other suffered remissions for three years and then recovered. Even in these selected cases it is therefore difficult to draw definite conclusions.

With regard to the seven patients who recovered, in four no focus of infection was demonstrated, in two the tonsils, and in one carious teeth were incriminated and removed.

\section{Cardiac complications}

Similarities between this disease and acute rheumatic fever raise the possibility of cardiac complications. In none of the old cases however was it possible to demonstrate a valvular lesion. It should be mentioned that R. Hutchison observed in one of his patients (included in this series) the development of a mid-diastolic murmur. This case was not traced in the recent follow-up enquiry. Adherent pericardium, on the other hand, is a recognized complication and was found in two of the autopsies and one of the surviving patients.

\section{Associated glandular enlargement}

Many of the cases surveyed showed marked glandular enlargement in the active stages (Still type), the axillary and cervical glands frequently being visible on inspection and the spleen sometimes extending to the umbilical level. This associated lymphoid hyperplasia appears to have no influence on the prognosis. Its presence or absence is determined by the age of onset. In cases beginning in the first four years of life lymphoid hyperplasia is rather the rule than the exception (table 1). In the older age groups it is progressively rarer. There is no period in life, however, when the onset of rheumatoid arthritis may not be associated with glandular enlargement.

TABLE 1.

The incidence of glandular enlargement in chronic pOlyarthritis in childhood.

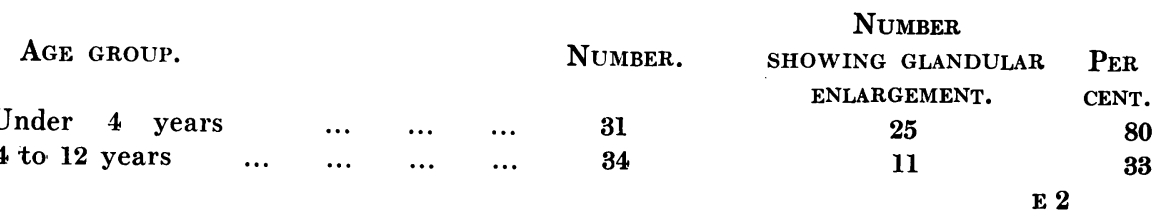




\section{Summary}

(1) In an effort to estimate the prognosis in chronic polyarthritis in childhood a consecutive series of sixty-nine patients have been surveyed and follow-up enquiries pursued.

(2) The disease appears to be be self limited, the average period of activity being nearly five years.

(3) In the pre-school period the incidence of the disease is highest and the mortality approaches thirty per cent. A fatal outcome is rare when the disease begins in the second half of childhood.

(4) The deaths occur in the first three years of activity. If this period is survived the danger to life is small.

(5) Only a rough estimation of the recovery rate can be deduced. Approximately one in four recover completely. Complete recovery is limited to those cases in which the disease becomes quiescent within three years.

(6) Very gross crippling is uncommon. Surviving cases can usually earn a livelihood.

Thanks are due to the medical staff of the Hospital for Sick Children for the facilities to make this enquiry and to Dr. Donald Paterson for valuable assistance. 